\title{
Three-dimensional spectrum of temperature fluctuations in stably stratified atmosphere
}

\author{
A. S. Gurvich and I. P. Chunchuzov \\ Oboukhov Institute of Atmospheric Physics, Moscow 119017, Russia
}

Received: 27 February 2008 - Revised: 24 April 2008 - Accepted: 16 June 2008 - Published: 28 July 2008

\begin{abstract}
A phenomenological model is proposed for a 3-D spectrum of temperature inhomogeneities generated by internal waves in the atmosphere. This model is a development of the theory based on the assumption that a field of Lagrangian displacements of fluid particles, induced by an ensemble of internal waves with randomly independent amplitudes and phases, is statistically stationary, homogeneous, axially symmetric in horizontal plane and Gaussian. For consistency of this model with measured spectra of temperature fluctuations in the stratosphere and mesosphere the additional assumption was introduced in to the model about the anisotropy of inhomogeneities to be dependent on their vertical sizes. The analytic expressions for both the 3-D and 1-D spectra are obtained. A model vertical wave number spectrum follows a -3 power law, whereas a horizontal spectrum contains two regions with a -3 slope, and the intermediate region with the slope between -1 and -3 depending on the rate of anisotropy decrease as a function of increasing sizes of the inhomogeneities. In the range of a few decades the model showed a good agreement with the results of measurements of the spectra in the troposphere, stratosphere and mesosphere.
\end{abstract}

Keywords. Meteorology and atmospheric dynamics (Middle atmosphere dynamics; Turbulence; Waves and tides)

\section{Introduction}

In this paper we propose a model of three-dimensional (3D) spectrum of the temperature fluctuations in stably stratified atmosphere with the vertical scales ranging from a few meters to several kilometers. Such scales are typical for atmospheric internal gravity waves (IGWs), which is known to form 3-D anisotropic temperature irregularities.

Correspondence to: I. P. Chunchuzov

(igor.chunchuzov@gmail.com)
When trying to parameterize the influence of IGWs on general atmospheric circulation we need a model of 3-D spectrum of IGWs in the atmosphere, since the parameterization models of wave drag are sensitive to the height dependence of this spectrum and to the vertical scale of wave breaking processes (Fritts and Alexander, 2003; McLandress, 1997; Hynes, 2005). The 3-D spectrum also determines the statistical properties of electromagnetic and acoustic waves propagating through the atmosphere (Tatarskii, 1971). The physical mechanisms explaining the observed forms of 1-D vertical and horizontal spectra of wind and temperature fluctuations were explored in a number of papers (VanZandt, 1982; Sidi et al., 1988; Dewan, 1997; Lindborg, 2006). However, the question about the form of a 3D spectrum of these fluctuations, which produces such 1-D spectra, remained open. It was shown in Chunchuzov (2002) (hereafter Ch02) that strongly anisotropic 3-D temperature and wind speed irregularities can occur in non-linear field of random internal waves in stably stratified atmosphere. Equilibrium 3-D spectrum obtained in this study results from the balance between the nonlinear wave energy transfer from wave sources with some characteristic vertical and horizontal scales towards lesser vertical scales (and simultaneously towards larger horizontal scales), and dissipation of the wave energy due to the wave breaking at certain scale. However, the $\mathrm{Ch} 02$ theory did not take into account a reverse influence of the turbulence, generated by breaking waves, on the wave spectrum itself. The emerging turbulent diffusion results in smoothing of the spatial gradients of the wave field, and affects characteristic vertical and horizontal scales of the field variations, and therefore its anisotropy.

The purpose of this study is to modify the Ch02 model, so that the influence of the diminishing vertical scale of the irregularities on their anisotropy is taken into account, and to compare a modified model with experimental data. 


\section{The theory}

An approximate expression for regularized 3-D spectrum of temperature fluctuations may be derived from the 3-D spectrum of vertical displacements induced by a random internal wave field (Ch02):

$$
\begin{aligned}
\Phi_{T}^{(0)}(\kappa) & =\frac{A_{0} T_{0}^{2} \omega_{\mathrm{BV}}^{4}}{g^{2}\left|\kappa_{z}\right|^{5}} \exp \left(-\frac{\kappa_{\perp}^{2}}{4 e_{0} \kappa_{z}^{2}}\right) R\left(\frac{\left|\kappa_{z}\right|}{\kappa_{*}}, b\right), \\
\kappa_{\perp}^{2} & =\kappa_{x}^{2}+\kappa_{y}^{2}, e_{0} \ll 1,
\end{aligned}
$$

where $\omega_{\mathrm{BV}}$ is Brunt-Väisälä (BV) frequency, $T_{0}$ - unperturbed temperature at a given altitude, $g$ - gravity acceleration, $e_{0}$ is a coefficient proportional to the variance of the horizontal gradient of a random Lagrangian field of the air particle's vertical displacements, and $A_{0}=\beta_{0} /\left(8 \pi e_{0}\right)$ is a nondimensional coefficient with $\beta_{0}=0.1 \div 0.3$.

The function $\Phi(\kappa)$ is considered to be a spectrum of locally homogeneous random field under the condition of existence of the corresponding structure function. The Eq. (1) results from Eq. (55a) in Ch02 after myltiplying Eq. (55a) by a factor $R$, which provides a regularization of the spectrum over the region of small wavenumbers $|\boldsymbol{\kappa}| \rightarrow 0$ for $\left|\kappa_{z}\right|<\kappa_{*}$; $\kappa_{*}$ is a characteristic vertical wave number of the spectrum. The introduced regularization factor $R(\zeta, b) \leq 1$ possesses the following properties: the ratio $R(\zeta, b) / \zeta^{b}$ is finite at $\zeta \rightarrow 0$ and $R(\zeta, b) \rightarrow 1$ for $\zeta>1 ; b>0$ is the regularization parameter.

Calculation from Eq. (1) of the one-side vertical wavenumber spectrum results in

$$
\begin{aligned}
V_{T}^{(0, v)}\left(\kappa_{z}\right) & =2 \iint \Phi_{T}^{(0)}(\kappa) d \kappa_{y} d \kappa_{x} \\
& =\beta_{0} \frac{T_{0}^{2} \omega_{\mathrm{BV}}^{4}}{g^{2} \kappa_{z}^{3}} R\left(\frac{\kappa_{z}}{\kappa_{*}}, b\right), \kappa_{z}>0,
\end{aligned}
$$

For $\beta_{0}=0.1 \div 0.3$ Eq. (2) is consistent with numerous experimental data reviewed by Fritts and Alexander (2003) (hereafter FA03). Also, calculation of the horizontal spectrum $V_{T}^{(0, h)}\left(\kappa_{y}\right)=\iint \Phi_{T}(\kappa) d \kappa_{y} d \kappa_{z}$ leads to the -3 power law decay, $V_{T}^{(0, h)}\left(\kappa_{y}\right) \sim\left|\kappa_{y}\right|^{-3}$, for $\left|\kappa_{y}\right|>\kappa_{*} \sqrt{2 e_{0}}$. Once the scaling $\kappa_{y}^{\prime}=\kappa_{y} / \sqrt{2 e_{0}}$ is applied, a one-side horizontal spectrum $V^{(0, h)}$ coincides with $V^{(0, v)}\left(\kappa_{y}^{\prime}\right)$ at $\kappa_{y}^{\prime}>\kappa_{*}$. The less is the value of $\sqrt{2 e_{0}}$, the more 3-D spectrum is stretched along $O \kappa_{z}$-axis and, accordingly, the irregularities are more flattened in vertical direction. This suggests that $1 / \sqrt{2 e}$ is a measure of anisotropy of the irregularities. Since the parameter $e_{0}$ is considered as constant (Ch02), Eq. (1) can be looked upon as a model of 3-D spectrum with constant anisotropy.

Measured horizontal spectra (Vinnichenko et al., 1980, Nastrom and Gage, 1985; Hostetler at al., 1991; Bacmeister et al., 1996; Cho et al., 1999; Lindborg, 1999) reveal the existence of spectral intervals where the dependence $V^{(0, h)}\left(\kappa_{y}\right)$ differs from $\left|\kappa_{y}\right|^{-3}$. In order to fit the model of the vertical and horizontal spectra, determined by 3-D spectrum, to the measurements, we modify Eq. (1), taking into account a possibility for the anisotropy of temperature irregularities to vary with their vertical sizes. We illustrate the concept of variable anisotropy $\eta$ by using the structure function $D_{T}(\delta \boldsymbol{r})=D_{T}\left(\delta r_{\perp}, \delta z\right)$ where $\delta r_{\perp}^{2}=\delta x^{2}+\delta y^{2}$. We assume that $\eta$ depends on vertical sizes of irregularities being a solution of the following equation: $D_{T}(0, \delta z)=D_{T}(\eta(\delta z) \delta z, 0)$. In other words the anisotropy depends on vertical sizes of irregularities, or using spectral language: $\eta=\eta\left(\kappa_{z}\right)$. It was shown by Gurvich (1997) that axis-symmetric 3-D spectrum with a varying anisotropy may produce the 1-D vertical and horizontal power spectra with different power indexes. Introducing a variable anisotropy into the model of 3-D spectrum, we should conserve the dependence $\left|\kappa_{z}\right|^{-3}$ for the vertical spectrum regardless of the form of $\eta=\eta\left(\kappa_{z}\right)$. Under our assumptions, the equation for 3-D temperature spectrum with variable anisotropy can be written as follows:

$\Phi_{T}(\kappa)=\frac{1}{2} \frac{A T_{0}^{2} \omega_{\mathrm{BV}}^{4} \eta\left(\kappa_{z}\right)^{2}}{g^{2}\left|\kappa_{z}\right|^{5}} \exp \left(-\frac{\kappa_{\perp}^{2} \eta\left(\kappa_{z}\right)^{2}}{2 \kappa_{z}^{2}}\right) R\left(\frac{\left|\kappa_{z}\right|}{\kappa_{*}}, b\right)$, $\kappa_{\perp}^{2}=\kappa_{x}^{2}+\kappa_{y}^{2}, \eta\left(\kappa_{z}\right) \geq 1$,

where $A$ is a numeric multiplier to be found from comparison (3) with the experimental data. For $\eta=$ const case, Eq. (3) is consistent with Eq. (1), taking into account the notation differences. The model (3) allows us to calculate the 1-D spectra $V_{T}\left(\kappa_{s} ; \alpha\right)$ for the arbitrary direction having an angle of $\alpha$ with respect to the horizontal plane

$$
\begin{aligned}
V_{T}\left(\kappa_{s} ; \alpha\right)= & \int_{-\infty}^{\infty} d \kappa_{x} d \kappa_{y} d \kappa_{z} \Phi_{T}\left(\kappa_{x}, \kappa_{y}, \kappa_{z}\right) \\
& \delta\left(\kappa_{y} \cos \alpha+\kappa_{z} \sin \alpha-\kappa_{s}\right)
\end{aligned}
$$

where $\delta(\zeta)$ is Dirac's delta function. The angles $\alpha=\pi / 2$ and $\alpha=0$ correspond to the horizontal and vertical spectra, respectively. The Eq. (4) is a compact expression for the integration along the plane, which is at a distance $\kappa_{s}$ from the coordinate origin and has the angle of $\pi / 2-\alpha$ with respect to the plane $\kappa_{x} O \kappa_{y}$.

To compare model (3) with the measurements, let us calculate vertical and horizontal spectra. The result of integration of Eq. (3) over $d \kappa_{x} d \kappa_{y}$ shows that vertical spectrum $V_{T}^{(v)}\left(\kappa_{z}\right)$ does not depend on $\eta\left(\kappa_{z}\right)$ and is similar to Eq. (2) at $\left|\kappa_{z}\right|>\kappa_{*}$ after replacing $\beta_{0}$ by the numeric coefficient $\beta=2 \pi A$. The dependence of $V_{T}^{(v)}\left(\kappa_{z}\right)$ on the regularizing factor $R$ is essential only at $\left|\kappa_{z}\right|<\kappa_{*}$.

Horizontal spectra calculation for model (3) results in the following equation

$$
V_{T}^{(h)}\left(\kappa_{y}\right)=\beta \sqrt{2 \pi} \frac{T_{0}^{2} \omega_{\mathrm{BV}}^{4}}{g^{2} \kappa_{y}^{3}} \int_{0}^{\infty} d \varsigma \frac{\eta\left(\kappa_{y} \varsigma\right)}{\varsigma^{-4}} R\left(\frac{\left|\kappa_{y}\right|}{\kappa_{*}} \varsigma, b\right)
$$




$$
\exp \left(-\frac{1}{2}\left(\frac{\eta\left(\kappa_{y} \varsigma\right)}{\varsigma}\right)^{2}\right), \kappa_{y}>0
$$

To compare Eq. (5) with the measurements, it is necessary to specify the functions $R(\zeta, b)$ and $\eta\left(\kappa_{z}\right)$. We will use the easiest version: $R(\xi ; b)=\xi^{b} /\left(\xi^{b}+1\right)$. Function $\eta\left(\kappa_{z}\right)$ will be chosen by using the available results of the measurements in the upper troposphere and lower stratosphere. The measurements conducted in Nastrom and Gage (1985) (hereafter NG85), show that $V_{T}^{(h)}\left(\kappa_{y}\right) \sim\left|\kappa_{y}\right|^{-3}$ for $2 \times 10^{-6}<\left|\kappa_{y}\right|<6 \times 10^{-5} \mathrm{rad} / m$. This suggests that $\eta\left(\kappa_{z}\right)$ has a constant value of the order of many tens, or hundreds, for wavenumbers $\kappa_{*}<\kappa_{z}<\kappa_{M}$ corresponding to the largestscale irregularities with the vertical sizes of about one kilometer or more. Measured angular dependence of the scattering cross-section of MST radar radiation (Doviac and Zrnic, 1984; Gurvich and Kon, 1993; Hermawan et al 1998) indicates that noticeable anisotropy $\eta_{0}>1$ still exists for smallscale stratospheric irregularities with vertical sizes of the order of a few meters. The analysis of star scintillation spectra observed from satellite through the Earth's stratosphere (Gurvich and Kan, 2003; Gurvich and Chunchuzov, 2003) leads to the same conclusion. To define $\eta\left(\kappa_{z}\right)$ for intermediate scales, we suggest the following interpolated formula

$$
\begin{aligned}
& \eta\left(\frac{\kappa_{z}}{\kappa_{w}}\right)=\eta_{0}\left(1+\left(\frac{\kappa_{w}}{\kappa_{M}}\right)^{p}\right), \text { if }\left|\kappa_{z}\right|<\kappa_{M} ; \\
& \eta\left(\frac{\kappa_{z}}{\kappa_{w}}\right)=\eta_{0}\left(1+\left|\frac{\kappa_{w}}{\kappa_{z}}\right|^{p}\right), \text { if }\left|\kappa_{z}\right|>\kappa_{M} ; p>0
\end{aligned}
$$

which will be tested using the complex of measurements. The parameter $p$ in Eq. (6) determines the rate at which the anisotropy decreases with decreasing vertical sizes of irregularities. As to the irregularities with the vertical sizes less than $1 / \kappa_{w}$, their anisotropy is small ( $\eta=$ const) and do not substantially change in the process of their evolution. Calculation of Eq. (5) using Eq. (6) results in the following asymptotic equations:

$$
\begin{aligned}
V_{T}^{(h)}\left(\kappa_{y}\right) \sim \beta \frac{T_{0}^{2} \omega_{\mathrm{BV}}^{4}}{g^{2}} \eta_{M}^{-2} \kappa_{y}^{-3}, \kappa_{*} / \eta_{M} \ll \kappa_{y} \ll \kappa_{M} / \eta_{M} \\
V_{T}^{(h)}\left(\kappa_{y}\right) \sim \beta \frac{T_{0}^{2} \omega_{\mathrm{BV}}^{4}}{g^{2}} \frac{2^{\frac{3+p}{2+2 p}} \sqrt{2 \pi}}{\pi(3+p)} \Gamma\left(\frac{5+3 p}{2+2 p}\right) \\
\quad \frac{\kappa_{y}^{-\frac{3+p}{p+1}}}{\eta_{0}^{\frac{2}{p+1}} \kappa_{w}^{\frac{2 p}{p+1}}}, \kappa_{M} / \eta_{M} \ll \kappa_{y} \ll \kappa_{w} / \eta_{0}
\end{aligned}
$$

$V_{T}^{(h)}\left(\kappa_{y}\right) \sim \beta \frac{T_{0}^{2} \omega_{\mathrm{BV}}^{4}}{g^{2}} \eta_{0}^{-2} \kappa_{y}^{-3}, \kappa_{y}>\kappa_{w} / \eta_{0}$.

The wider the interval of acceptable values of $\kappa_{y}$, the better approximation is provided by Eqs. (7-9). These equations

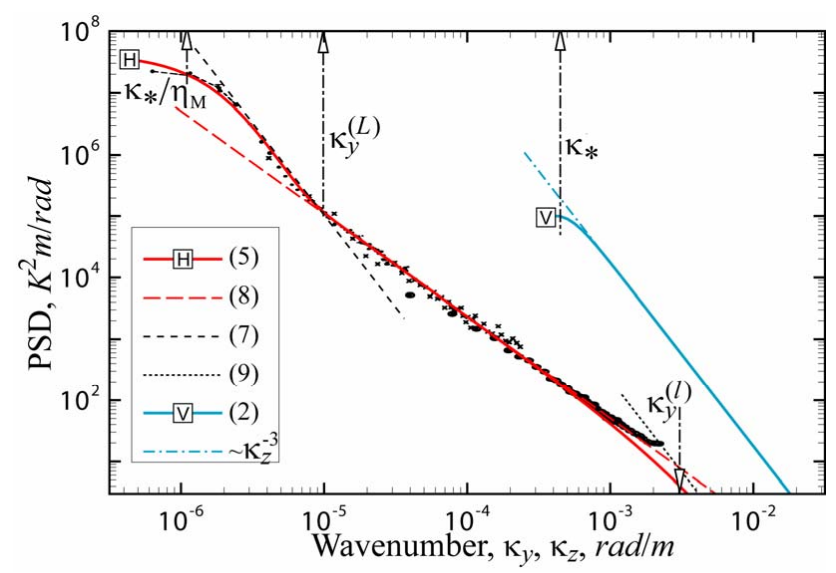

Fig. 1. Comparison of the modeled horizontal (H) and vertical (V) spectra with the measurements (Nastrom and Gage, 1985). The dots depict the measurements. The equations used to calculate spectral densities (solid curves) and the asymptotic power-law functions (dashed straight lines), are referred to in the legend. Vertical arrows indicate the abscissas for the intersection points of the curves (7) with (8) (labeled by $\kappa_{y}^{(L)}$ ), and (8) with (9) (labeled by $\kappa_{y}^{(l)}$ ).

simplify the analysis of Eq. (5) and demonstrate that the horizontal spectrum may consist of the three consecutive intervals, two of which have a power of -3 , while the power for the intermediate range $(8)$ is $-(3+p) /(p+1)$. The latter can take the values from -3 to -1 .

\section{Comparison with measurements}

The validation of Eqs. (3) and (5) was made using measured 1-D horizontal spectra (NG85; Hostetler at al., 1991; Bacmeister et al., 1996). The data presented in NG85 were gathered during several thousands of flights and were strongly verified by the measurements (Cho et al., 1999; Lindborg, 1999). The dots in Fig. 1 show the estimated values of the horizontal spectrum from NG85. The latter, unfortunately, does not provide all the characteristics of the measurement conditions. Thus, additional considerations are required to choose the missing parameters of the model spectra, which are necessary for calculations. The values of $g=9.81 \mathrm{~m} / \mathrm{s}^{2}$ and $T_{0}=220 \mathrm{~K}$ are beyond questioning. It follows from Eqs. (7-9) that the behavior of 1-D horizontal spectrum at wavenumbers $\kappa_{y}>\kappa_{*} / \eta_{M}$ is not very sensitive to the regularization parameter $b$, therefore the value of $b=5$ has been chosen. The BV frequency $\omega_{\mathrm{BV}}$ was assumed to be $\omega_{\mathrm{BV}}=0.02 \mathrm{rad} / \mathrm{s}$. The value $\kappa_{y} \approx 10^{-6}$ corresponding to the range, where spectral density measured by NG85 becomes saturated, represents the estimate of the initial characteristic horizontal wavenumber $\kappa_{*} / \eta_{M}$. The value $\kappa_{y}=10^{-5} \mathrm{rad} / \mathrm{m}$, corresponding to the inflection interval of the measured spectrum, became the basis for the initial estimate of $\kappa_{M}$. The measurements in Hermawan et al. (1998) indicate that the 


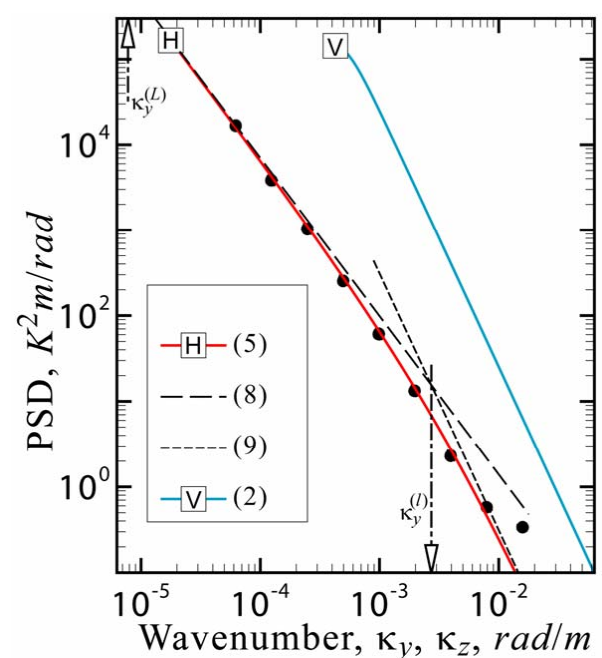

Fig. 2. Comparison of the modeled horizontal (H) and vertical (V) spectra with the measurements (Bacmeister et al., 1996). The dots depict the measurements. The equations used to calculate spectral densities (solid curved lines) and the asymptotic power-law functions (dashed straight lines), are referred to in the legend. Vertical arrows indicate the characteristic wavenumbers.

possible value of $\eta_{0}$ is about ten. Final adjustment of the parameters in Eqs. (5) and (6) was made using a trial-anderror method. Characteristic wavenumbers were found to be $\kappa_{*}=4.47 \times 10^{-4}, \kappa_{M}=1.5 \times 10^{-3}, \kappa_{w}=10^{-2} \mathrm{rad} / \mathrm{m}$. The values $p=2$ and $\eta_{0}=9$ was found to provide the best fit to the measured spectra. The obtained value $\beta=0.22$ is in a good agreement with the numerous measurements of the vertical spectra (FA03), therewith the value $\eta_{M}=409$ is derived. Figure 1 shows the model spectrum $V_{T}^{(h)}\left(\kappa_{y}\right)$, calculated from Eq. (5), which depicts all the details of the NG85 measurements. The vertical spectra are not presented by NG85, but the model (3) allows one to obtain them, given the parameters calculated from the measurements of horizontal spectrum. The vertical spectrum $V_{T}^{(v)}\left(\kappa_{z}\right)$, calculated accordingly to Eq. (2), is also shown in Fig. 1. Relations (7-8) depict in Fig. 1 the two power-law intervals of the measured spectrum with power indexes -3 and $-5 / 3$. Note that the model is in good agreement with the measurements throughout the wavenumber range of more than three decades. The $k_{y}^{-3}$ shape of the low-wavenumber part of the NG85 horizontal spectrum may be interpreted by a theory of quasi-geostrophic turbulence with an enstrophy cascade from longer to shorter horizontal wavelengths (see, for instance, Charney, 1971; Lindborg, 1999, 2006). At the same time, our model suggests that internal wave-wave interactions may also contribute to the same large-scale part of the spectrum (given by Eq. 7) by nonlinear wave energy transfer from shorter horizontal scales. As to the third power-law range (Eq. 9) with the power index of -3 , the spatial resolution in the NG85 measurements appears to be not good enough to investigate the spectrum at such short scales.
The dots in Fig. 2 depict the measurements of the spectra (Bacmeister, 1996), conducted at the altitude of about $20 \mathrm{~km}$. Wavenumber range is expanded by almost one order towards large wavenumbers, compared to the NG85 data. However, this range is limited from below by the value $\left|\kappa_{y}\right| \approx 6 \times 10^{-5} \mathrm{rad} / \mathrm{m}$, and this complicates the process of choosing the values of $\kappa_{*}$ and $\kappa_{M}$, since they appear to be below the limiting value of $\left|\kappa_{y}\right|$. It follows from the Eqs. (7-9) that the behavior of $V_{T}^{(h)}\left(\kappa_{y}\right)$ at $\kappa_{y}>\kappa_{M} / \eta_{M}$ has to depend only slightly on $\kappa_{*}$ and $\kappa_{M}$. So, to conduct the comparison, we have taken the same values for these parameters as those used above. The value $\omega_{\mathrm{BV}}=0.022 \mathrm{rad} / \mathrm{s}$ was taken accordingly to the data obtained in Bacmeister (1996, Fig. 11). With previously chosen $T_{0}=217 \mathrm{~K}, \eta_{0}=9$ and $\beta=0.22$, we had to define the parameter $p$, wavenumber $\kappa_{w}$, and $\beta$. The trial-and-error method gave the values: $p=1.35$ and $\kappa_{w}=9.5 \times 10^{-3} \mathrm{rad} / \mathrm{m}$. Given these parameters, $\eta_{M}=263$ and $(3+p) /(p+1)=1.85$. The last is close to the value of -1.67 obtained by NG85. The measurements in Bacmeister (1996) fall into the range of transition from the asymptotic dependence to the power-law dependence $\sim \kappa_{y}^{-3}$. The agreement between the model and measurements is quite satisfactory up to $\kappa_{y}=8 \times 10^{-3} \mathrm{rad} / \mathrm{m}$, above which the effects of buoyancy forces on spectral power law decay was suggested to become negligibly small (Bacmeister, 1996).

The results of synchronized aircraft-based measurements of vertical and horizontal spectra in the layer between 80 and $105 \mathrm{~km}$ were published in Hostetler et al. (1991). The spectra obtained from these measurements and the calculated model spectra, which take into account the effects of averaging over the time duration of laser pulse and the time interval of the accumulation of scattered photons, are shown in Fig. 3. Using Eq. (2), we obtained an estimate $\beta=0.28$ from the measured vertical spectrum, for which we have chosen the parameters $g=9.5 \mathrm{~m} / \mathrm{s}^{2}, T_{0}=160 \mathrm{~K}$ and $\omega_{\mathrm{BV}}=0.025 \mathrm{rad} / \mathrm{s}$ so as to match the atmosphere model CIRA-61. We point out that the deviation of the model spectrum from power-law dependence $\sim \kappa_{z}^{-3}$ at large $\kappa_{z}$ results from the time averaging, accounted for in calculations. The remaining parameters were adjusted, as previously, using trial-and-error method. This procedure was complicated by the fact that the range of wavenumbers for the published results of the horizontal spectra measurements does not embrace all possible characteristic wavenumbers. The best agreement between the model and measurements has been gained for the following parameters: $\kappa_{*}=\kappa_{M}=3.9 \times 10^{-4} \mathrm{rad} / \mathrm{m}, b=3.5, \eta_{0}=15$, and $p=0.6$. For these parameters, the maximum value of the anisotropy coefficient $\eta_{M}=135$. Let us dwell on the parameter $p$ which leads to the power index $-(3+p) /(1+p)=-2.25$. This value is sufficiently close to the estimates for horizontal spectrum presented in Hostetetler et al. (1994).

The oblique spectra measured by using dive flights with $\alpha=0.1 \mathrm{rad}$ and the horizontal spectra are published in Gurvich and Kukharez (2008). These measurements were 

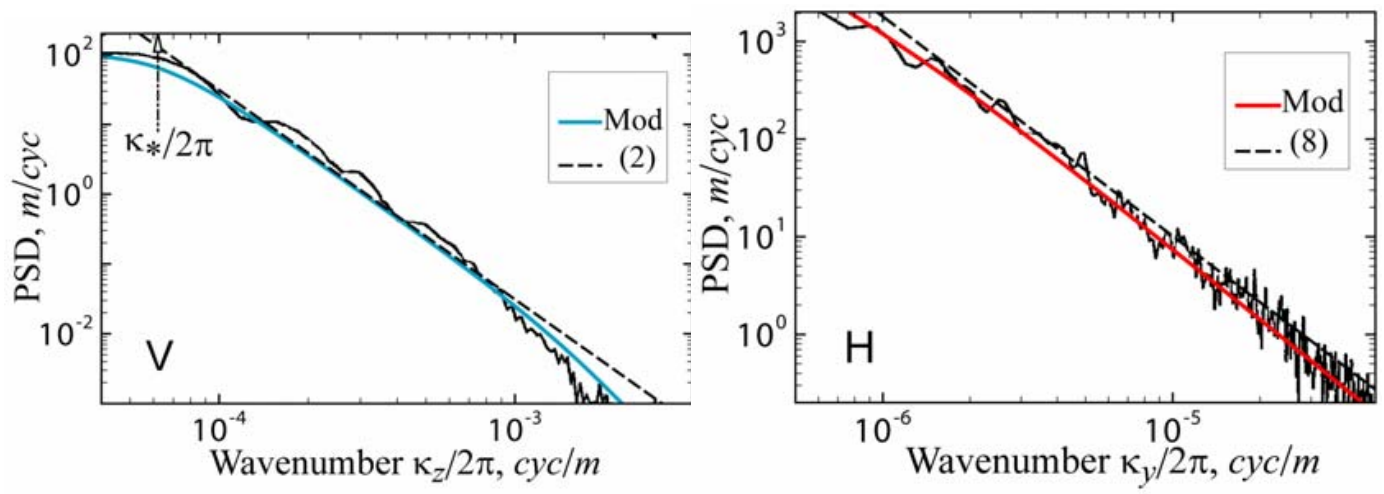

Fig. 3. Comparison of the model with the measurements (Hostetler et al., 1991). Vertical (V) and horizontal (H) spectra of the relative density fluctuations are shown. Black solid line: measurements; Mod: model; the equations used to calculate asymptotic power-law functions are referred to in brackets.

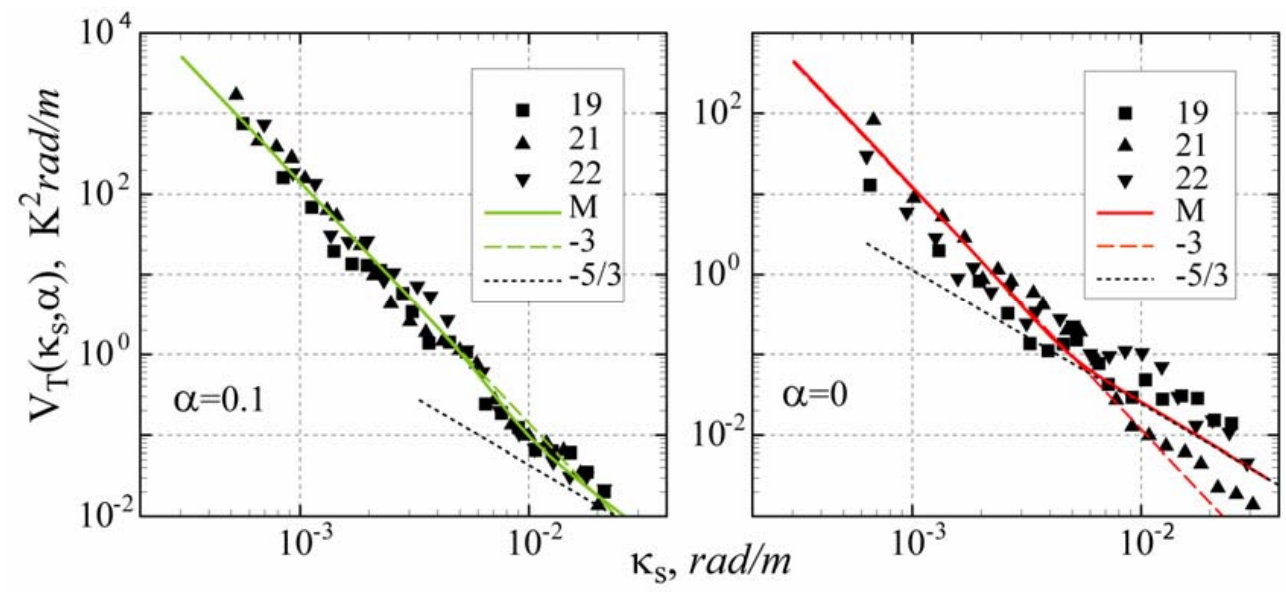

Fig. 4. Comparison of the model with the measurements of Gurvich and Kukharez (2008). Left panel is oblique spectra ( $\alpha=0.1)$, right panel is horizontal $(\alpha=0)$ spectra. M is model calculated by using Eq. (4), dot and dash lines is asymptotic power law, symbols are experimental estimations, 19-22 are flight days.

performed in 1988 on 19, 21, and 22 July. The three pairs of the flights were performed through stable stratified layer of the troposphere between altitudes 2.5 and $7.5 \mathrm{~km}$. The measurements of the mean vertical gradient of temperature allowed us to estimate the values of $\omega_{\mathrm{BV}}$, which were close to $0.01 \mathrm{rad} / \mathrm{s}$. The plots of Fig. 4 show the measured oblique and horizontal spectra. Also shown in the plots are the model spectra, which were calculated under mean flight conditions, with the mean temperature $T_{0}=268 \mathrm{~K}$. We used the Eq. (4) for calculating of the model spectra., for which the best fitting parameters were: $\kappa_{M}=4.8 \times 10^{-2}, \kappa_{w}=1.7 \times 10^{-1} \mathrm{rad} / \mathrm{m}$, $\eta_{0}=1.6, \beta=0.25, p=2$.For these parameters the maximum value of the anisotropy $\eta_{M}=22$. The plots of Fig. 4 show the agreement between measured and model spectra, especially for the case of oblique spectrum.

\section{Summary}

The model of a 3-D temperature spectrum $\Phi_{T}(\boldsymbol{\kappa})$ presented in this paper is an outgrowth of the theoretical study (Ch02) added by a variable anisotropy hypothesis. This model gives a first consistent description of all possible 1-D spectra in the stably stratified atmosphere. Using one set of the main parameters of the model the obtained 3-D spectrum produces the vertical, horizontal, and oblique spectra that are in a good agreement with the observations (Nastrom and Gage, 1985; Hostetler at al., 1991; Bacmeister et al., 1996; Gurvich and Kukharez, 2008). When taking into account the dependence of the irregularities' anisotropy on their size the model developed here was capable of accounting for both the slopes and the amplitudes of the measured vertical and horizontal spectra over the wide range of wavenumbers. 
Acknowledgements. This work was supported by RFBR grants: 0605-64354, 07-05-91555 and 06-05-64229.

Topical Editor U.-P. Hoppe thanks two anonymous referees for their help in evaluating this paper.

\section{References}

Bacmeister, J. T., Eckermann, S. D., Newman, P. A., Lait, L., Chan, K. R., Loewinstein, M., Proffit, M. H., and Gary, B. L.: Stratospheric horizontal wavenumber spectra of winds, potential temperature, and atmospheric tracers observed by high-altitude aircraft, J. Geophys. Res., 101(D5), 9441-9470, 1996.

Charney, J. G.: Geostrophic turbulence, J. Atmos. Sci., 28, 10871095, 1971.

Cho, J. Y. N., Chu, Y., Newel, R. E., Anderson, B. E., Barrick, J. D., Gregory, G. L., Sachse, G. W., Carroll, M. A., and Albercook, G. M.: Horizontal wavenumber spectra of winds, temperature, and trace gases during the Pacific Exploratory Missions: 1. Climatology, J. Geophys. Res., 104(D5), 5697-5716, 1999.

Chunchuzov, I. P.: On the high-wavenumber form of the Eulerian internal wave spectrum in the atmosphere, J. Atmos. Sci., 59, 1753-1772, 2002.

Dewan, E. M.: Saturated Cascade Similitude Theory of Gravity Wave Spectra, J. Geophys. Res., 102, 29 799-29 817, 1997.

Doviac, R. J. and Zrnic, D. S.: Reflection and scatter formulas for anisotropic air, Radio Sci., 19, 325-336, 1984.

Fritts, D. C. and Alexander, M. J.: Gravity Wave Dynamics and Effects in the Middle Atmosphere, Rev. Geophys., 41(1), 1003, doi:10.1029/2001RG000106, 2003.

Gurvich, A. S.: A heuristic model of three-dimensional spectra of temperature irregularities in the stably stratified atmosphere, Ann. Geophys., 15, 856-869, 1997, http://www.ann-geophys.net/15/856/1997/.

Gurvich, A. S. and Chunchuzov, I. P.: Parameters of the fine density structure in the stratosphere obtained from spacecraft observations of stellar scintillations, J. Geophys. Res., 108(D5), 4166, doi:10.1029/2002JD002281, 2003.

Gurvich, A. S. and Kan, V.: Structure of Air Density Irregularities in the Stratosphere from Spacecraft Observations of Stellar Scintillation: 2. Characteristic Scales, Structure Characteristics, and Kinetic Energy Dissipation, Izvestiya, Atmos. Oceanic Phys., 39, 311-321, 2003.
Gurvich, A. S. and Kon, A. I.: Aspect sensitivity of radar returns from anisotropic turbulent irregularities, J. Electromagn. Waves Appl., 7, 1343-1353, 1993.

Gurvich, A. S. and Kukharez, V. P.: Horizontal and oblique temperature spectra in the stably stratified troposphere, Izvestiya, Atmos. Oceanic Phys., 44(6), accepted, 2008.

Haynes, P.:, Stratospheric Dynamics, Ann. Rev. Fluid Mech., 37, 263-93, doi10.1146/annurev.fluid.37.061903.175710, 2005.

Hermawan, E., Tsuda, T., and Adachi, T.: MU radar observations of tropopause variations by using clear air echo characteristics, Earth Planets Space, 50, 361-370, 1998.

Hostetler, C. A. and Gardner, C. S.: Observations of horizontal and vertical wave number spectra of gravity wave motions in the stratosphere and mesosphere over the mid-Pacific, J. Geophys. Res., 99(D1), 1283-1302, 1994.

Hostetler, C. A., Gardner, C. S., Vinsent, R. A., and Lesicar, D.: Spectra of Gravity Wave Density and Wind Perturbations Observed during ALOHA-90 on the 25 March Flight between Maui and Christmas Island, Geophys. Res. Lett., 18(7), 1325-1328, 1991.

Lindborg, E.: Can the atmospheric kinetic energy spectrum be explained by two-dimensional turbulence?, J. Fluid. Mech., 388, 259-288, 1999.

Lindborg, E.: The energy cascade in a strongly stratified fluid, J. Fluid Mech., 550, 207-242, 2006.

McLandress, C.: Sensitivity Studies using the Hines and Fritts gravity wave drag parameterizations, in: Gravity Wave Processes, Their Parameterization in Global Climate Models, edited by: Hamilton, K., Berlin, SpringerVerlag, 245-257, 1997.

Nastrom, G. D. and Gage, K. S.: A Climatology of Atmospheric Wavenumber Spectra of Wind and Temperature Observed by Commercial Aircraft, J. Atmos. Sci., 42(9), 950-960, 1985.

Sidi, C., Lefrere, J., Dalaudier, F., and Barat, J.: An Improved Atmospheric Buoyancy Wave Spectrum Model, J. Geophys. Res., 93, 774-790, 1988.

Tatarskii, V. I.: The Effects of the Turbulent Atmosphere on Wave Propagation, 417 pp., US Dep. of Commer., Springfield, Va, 1971.

VanZandt, T. E.: An Universal Spectrum of Buoyancy Waves in the Atmosphere, Geopys. Res. Lett., 9, 575-578, 1982.

Vinnichenko, N. K., Pinus, N. Z., Shmeter, S. M., and Shur, G. N.: Turbulence in the Free Atmosphere, 2nd ed., 310 pp., Plenum, New York, 1980. 